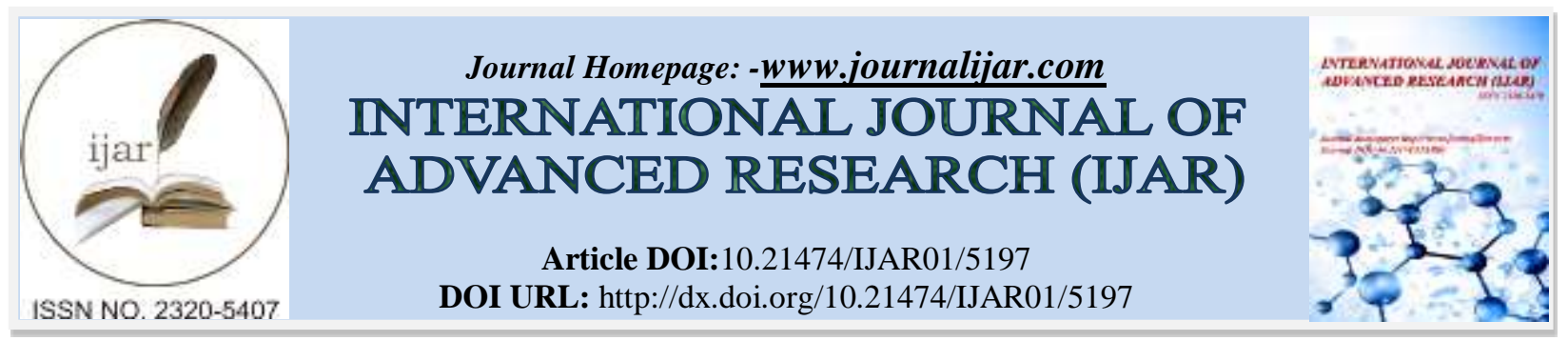

RESEARCH ARTICLE

\title{
CRISPR KNOCKOUT OF TAX1BP3 REDUCES EXPRESSION OF P53 IN HUMAN PAPILLOMA NEGATIVE AND HUMAN PAPILLOMA POSITIVE CERVICAL CARCINOMA CELLS.
}

\section{Mona El Helaly El Sherbeny ${ }^{1}$, Hussein Abdelaziz Abdalla ${ }^{1}$, El Said Mohamed Abd El Hady ${ }^{2}$, Ian Noel}

Hampson $^{3}$,Anthony.W.Oliver ${ }^{3}$, Souad Mohamed Abo Azma ${ }^{1}$, and Muhamad Muhamad Said ${ }^{1}$.

1. Department of Medical Biochemistry, Faculty of Medicine, Mansoura University, El Gomhouria Street, Dakahlia Governorate, Egypt.

2. Department of Obstetrics and Gynecology, Faculty of Medicine, Mansoura University, El Gomhouria Street, Dakahlia Governorate, Egypt.

3. School of Cancer and Imaging Science, Research Floor, St Mary's Hospital, Hathersage Road, Manchester M13 0JH. UK.

\section{Manuscript Info}

\section{Manuscript History}

Received: 17 June 2017

Final Accepted: 19 July 2017

Published: August 2017

Key words:-

Tax 1binding protein 3; human papillomavirus (HPV) 16 E6; p53 ; CRISPR/Cas9.

\section{Abstract}

Background: The PDZ protein Tax 1 binding protein 3 (Tax1BP3) is unique since, unlike other PDZ proteins, it contains a single rather than multiple PDZ domains. Previous work has shown Tax1BP3 is a target of oncoproteins from two separate human tumor viruses and it is known to regulate Rho and wnt/ $\beta$-catenin signaling. Most importantly Tax1BP3 has been shown to promote degradation of the p53 protein in glioma cells although it is not clear whether it performs this same function in tumor virus positive cell types .

Methods: The cervical cancer cell lines C33A (HPV negative), C33AE6 (HPV16 E6 transfected) and CaSki (HPV16 positive) were stably transfected with a CRISPR/Cas9 Tax1BP3 knockout construct. Levels of Tax1BP3 mRNA were initially determined in puromycin resistant monoclonal cells by RT-PCR. Tax1BP3 and p53 protein levels were then analyzed by a combination of western immunoblotting and flow cytometry.

Results: Both bi and monoallelic Tax1BP3 targeted cell lines were isolated for all three cell types which showed either partial or complete silencing of Tax1BP3 expression. Irrespective of cell type, all cells which had reduced or absent Tax1BP3 expression showed much lower levels of p53 protein than control cells..

Conclusion: Unlike previous findings with glioma cells, the presented data showed that reduced levels of Tax1BP3 directly correlated with reduced levels of p53 protein expression. Furthermore, this effect was seen in cells whether or not they expressed mutant (C33A) or wild type (CaSki) p53 or full or partial HPV genome (CaSki and C33AE6). These results suggest that Tax1BP3 regulates p53 protein levels in HPV positive and negative cells by a novel and non-canonical means. 


\begin{abstract}
Abbreviation:-
Tax1BP3: Tax 1binding protein 3

CRISPR/Cas9:Clustered Regularly Interspaced Short Palindromic Repeats/CRISPR associated nuclease 9

HPV16: human papilloma virus type 16
\end{abstract}

\title{
Introduction:-
}

Tax1BP3(Tax 1binding protein 3), also known as Tax interacting protein 1 (Tip-1) or glutaminase interacting protein (GIP) is a multifunctional protein targeting many proteins in diverse signaling pathways involved in cancer and other disease (Mohanty et al., 2015). Tax1BP3 consists of single PDZ domain which makes Tax1BP3 unique since other PDZ proteins possess multiple PDZ domains (Wang et al., 2014).

It has been previously shown that Tax1BP3 interacts with the human T-cell leukemia virus type 1 Tax (Rousset et al., 1998), and HPV16 E6 oncoproteins (Hampson et al., 2004). Most significantly, unlike other E6 protein targets (Ainsworth et al., 2008), Tax1BP3 was not degraded by its interaction with E6 which led to speculation that this interaction may produce an oncogenic gain-of-function (Hampson et al., 2004). In this regard Tax1BP3 has also been shown to interact with the guanidine exchange factor ARHGEF16 which resulted in E6-dependent activation of the Rho protein cdc42 in cervical carcinoma cells which is known to promote malignant transformation and progression (Oliver et al., 20011) Tax1BP3 is also known to regulate both Rho-A (Hampson et al., 2004) and $\beta$ catenin/wntsignallig (Kanamori et al., 2003) which are both considered as crucial pathways involved in neoplastic transformation. Elevated Tax1BP3 was also detected in invasive breast cancer combined with xenograft studies which demonstrated its ability to regulate tumor cell motility and adhesion (Han et al., 2012).

Other work has shown that Tax1BP3 may play a role in the development of brain tumors. For example it is known that it interacts with the DTX1(Deltex ubiquitin E3 ligase 1),STAU1(staufen double stranded RNA binding protein 1), glutaminase L and BAL2(2,3 dimercaptophenol) proteins which are involved in normal brain function and yet abnormalities in their function have been linked to the formation of brain tumors (Zencir et al., 2013). Moreover, Tax1BP3 has also been shown to interact with the guanidine exchange factor ARHGEF7 and to regulate Rho signaling in gliobalstome cells and anti Tax1BP3 antibodies have been shown to control tumor growth in tumorxenografts (Wang et al., 2014,Yan et al., 2016). Furthermore, most relevant to the current study, Tax1BP3 has been shown to promote the ubiqutination and degradation of the tumor suppressor p53 is glioma cell lines (Han et al., 2012) . CRISPR/Cas 9 (Clustered Regularly Interspaced Short Palindromic Repeats/CRISPR associated nuclease 9) is RNA-guided genome editing tool in which 20 nt guide RNA sequence direct the cas9 to the target gene by base pairing, resulting in site specific DNA double -strand breaks(DSBs) which subsequently repaired by non homologous end joining (NHEJ) pathway or homology directed repair (HDR) pathway result in disruption of the target gene (Ebina et al., 2013, Cong et al., 2013).

CRISPR/Cas 9 system is efficient, easy to design and simple to use in comparison to other programmable nucleases such as zinc finger nuclease (ZFNs) and transcription activator like effector nucleases (TALENs) which are difficult and time consuming to design(Gaj et al., 2013, Mali et al., 2013) .

With these advantages CRISPR/Cas 9 system has become an efficient and convenient tool for genome editing and has been used to study many cancer associated genomic mutations (Zhen et al., 2014, Lin et al., 2014).

In light of this work, we now present a study on the effects of CRIPSR mediated gene knock out of Tax1BP3 on p53 levels in cultured HPV positive and negative cervical carcinoma cell lines expressing wild type and mutant p53 in the presence of the full or partial HPV16 genome.

\section{Material and methods:- Cell lines and Cell culture condition}

Three cervical cancer cell lines C33A (HPV negative), C33AE6 (HPV16 E6 transfected) and CaSki (HPV16 positive) were grown in Roswell Park Memorial Institute medium (RPMI) -1640 (Invitrogen Ltd.) supplemented with $10 \%$ fetal bovine serum (FBS) and $2 \mathrm{mM}$ L-glutamine (Sigma-Aldrich). 


\section{CRISPR/Cas9 construct, stable gene transfection and Tax1BP3 silencing}

Genome-Wide knockout kit using CRISPR /Cas9 gene knockout/ knock in kit specific for Tax1BP3 was obtained from (Origene) was used the kit used to knockout Tax1BP3 gene through gene specific guide vector and knock in a functional cassette containing GFP (green fluorescent protein) gene and puromycin resistant gene by using a donner vector . Tax1BP3 Single guide RNA (sgRNA) was cloned into pCas guide vector and were designed to target the amino-terminal region of the Tax1BP3 open reading frame (ORFs)Tax1BP3sgRNA Sequence: CGTTTCCCGCTCTGCGAGG.

A negative scramble gRNA control cloned into pCas guide vector was also provided. All cell lines were stably transfected with guide and donner vector provided with knockout/knock (origine) according to manufacturer's instruction kit and lipofectamine 2000 (Invitrogen) was used as a transfection reagent .Using the identical procedure and culture condition all cell lines were stably transfected with scramble control and donnervector.Then all cells were subcultured for 7 times before monoclonal selection with puromycin the optimal puromycin concentration was $(1 \mu \mathrm{g} / \mathrm{ml})$ for c33A and C33AE6 while $(0.2 \mu \mathrm{g} / \mathrm{ml})$ for caski cell line .

\section{RNA extraction and reverse transcription polymerase chain reaction (RT-PCR)}

Total RNA was extracted using Ambion kit (Invitrogen). $1 \mu \mathrm{g}$ of total RNA were subjected to a reverse transcription (RT) using TetrocDNA Synthesis Kit (Bioline) as recommended by the manufacturer.1 $\mu 1$ of RT reaction mixture were subjected to PCR. For Tax1BP3 mRNA amplification, the forward primer 5' GTACTCTGCTGCCGGCTTCT-3'and reverse primer, 5'-TCACCAAGCGCTCGGAGGAG-3' were used with thermal cycles set at the condition as follow; $94^{\circ} \mathrm{C}$ for $2 \mathrm{~min}, 35 \mathrm{cycles}$ of $94^{\circ} \mathrm{C} 30 \mathrm{sec}, 55^{\circ} \mathrm{C} 30 \mathrm{sec}, 72^{\circ} \mathrm{C} 30 \mathrm{sec}$, followed by $72{ }^{\circ} \mathrm{C}$ for $5 \mathrm{~min}$.

\section{western blot analysis and antibodies}

western blot experiments were carried out according to standard protocols and were developed by using the enhanced chemiluminescence detection system (GE Healthcare Lifesciences, Buckinghamshire, UK).the following primary antibodies were used The TAX1BP3 mouse monoclonal primary antibody ( Abgent), the mouse monoclonal primary antibody p53 ab-6 (calbiochem), loading control were mouse monoclonal to GAPDH ab9484 (Abcam). The secondary antibody was polyclonal rabbit antimouse HRP conjugated for western applications(DAKO) .

\section{Flow cytometric analyses}

Cells were grown in T-25 flasks (Corning, US) to a confluence of $70 \%$. Cells were harvested and counted, $1 \times 10^{6}$ cells were centrifugated then resuspended in $5 \mathrm{ml}$ of $1 \mathrm{x}$ PBS at $4{ }^{\circ} \mathrm{C}$ and washed by centrifugation 5 minutes. The cell pellet was then fixed with $1 \mathrm{ml}$ of ice-cold $70 \%$ Ethanol and stored overnight at $4{ }^{\circ} \mathrm{C}$. The next day, cells were centrifugatedand The supernatant was aspirated and the anti p53 antibody diluted 1:1000 (Promega Ltd UK) was added to each sample at $10 \mathrm{mg} / \mathrm{ml}$. The samples were then stored for 1 hour at $4{ }^{\circ} \mathrm{C}$. After this incubation period, samples were centrifugated for 5 minutes at $4{ }^{\circ} \mathrm{C}$, washed with ice-cold $1 \times \mathrm{xBS}$, then centrifugated again for 5 minutes at $4{ }^{\circ} \mathrm{C}$. The 1 x PBS was aspirated until only $300 \mu l$ remained over each pellet. These were then resuspended and transferred to a Falcon flow cytometry tube (Jencons Ltd UK). Samples were analysed on a BD FACSCanto ${ }^{\mathrm{TM}}$ Flow Cytometer.

\section{Results:-}

\section{CRISPR/Cas9-Mediated Targeted Disruption of Tax1BP3gene}

After stable transfection cells were subjected to puromycin selection for 2 weeks until monoclones with stably integrated plasmid were appeared then we have isolated stable monoclonones totally 50 monoclones form each cell line.

The presented result show that CRISPR/Cas can efficiently inactivate Tax1BP3 in HPV positive and negative cervical cancer monoclones and we were successfully able to isolate completely silenced Tax1BP3 (biallelic knockout) monoclones from all cell lines in addition to many monoclones with partial Tax1BP3 knockout (monoallelic knockout). 


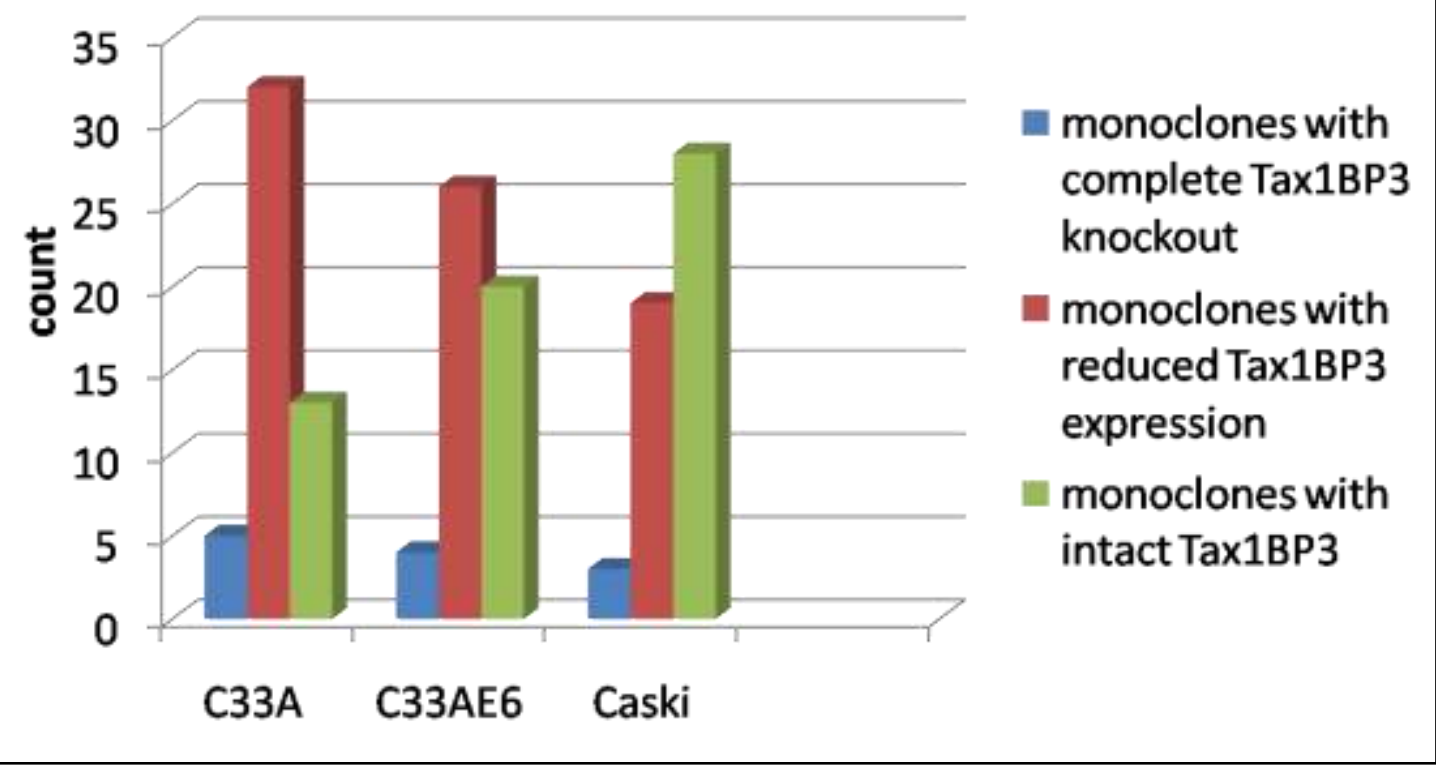

(Fig 1) statistical analysis Tax1BP3 expression of 50 stably transfected monoclones isolated after CRISPR transfection of C33A, C33AE6 and caski cell lines using Pearson's Chi-square or fisher exacttest.The number of monoclones with affected Tax1BP3 relative to those with intact Tax1BP3 was with high significance $<0.001$.

\section{Tax1BP3 disruption led to absence of Tax1BP3 mRNA and protein}

RT-PCR results confirm absence of Tax1BP3 after CRISPR/Cas application After antibiotic selection RNA were extracted from c33a and c33aE6 monocoles with stably integrated plasmid then RT-PCR was done to analyze Tax1BP3 RNA expression in stable monoclones. The presented results show the ability of CRISPR/CAS9 perturb Tax1BP3 gene and consequence absence of Tax1BP3RNA in RT-PCR analysis (figure 2).

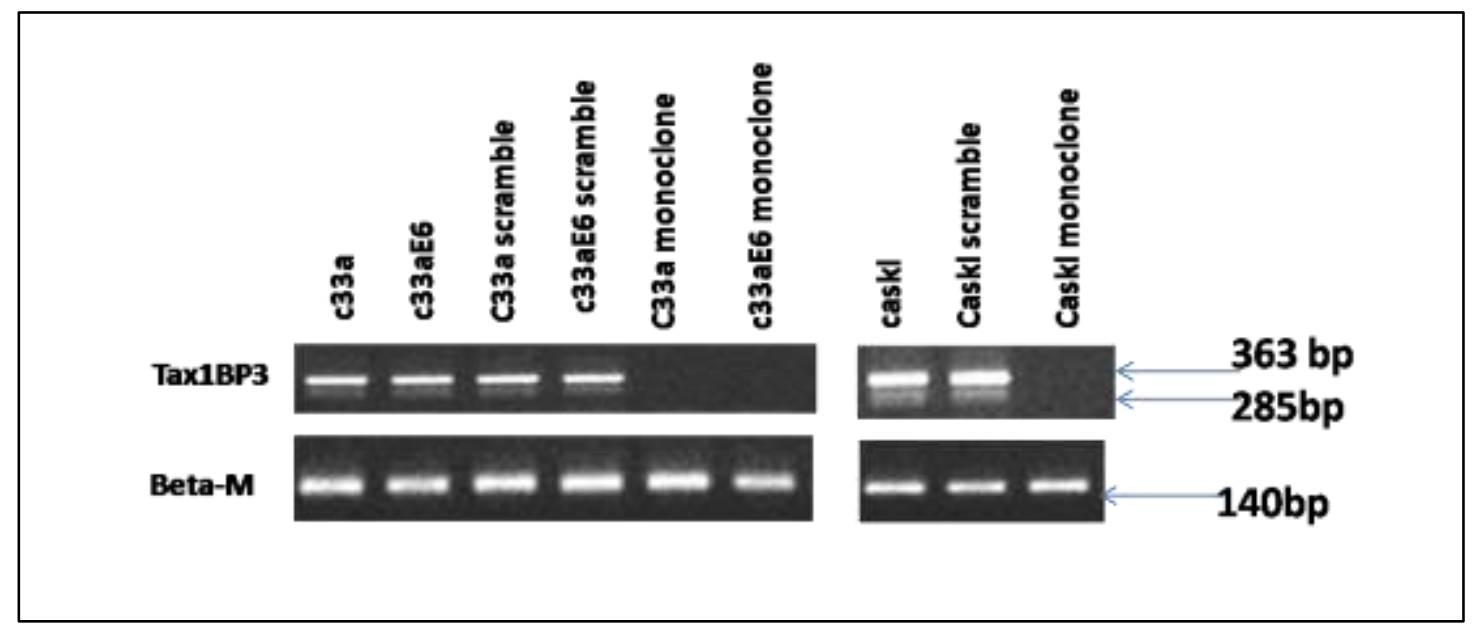

(Fig 2) RT-PCR analysis showing absence of Tax1BP3 RNA After CRISPR/Cas mediated Tax1BP3 knockout.

Western blot confirms the effect of CRISPR/Cas system on Tax1BP3 protein level protein were extracted from c33a and c33aE6 and caski stable monocolones then western blot analysis was done to investigate Tax1BP3expression. monoclones with complete knockout show complete absence of protein signal while those monoclones with partial knockout show reduced expression signals(figure 3,4,5). 


\section{Tax1BP3 knockout markedly reduce p53 expression level in HPV positive and HPV negative monclones}

Protein lysate was extracted from C33A, C33aE6 and caskimonoclones with complete Tax1BP3 knockout to asses p53 expression level using western blot and interestingly p53 expression levels were markedly reduced in comparison to their controls, taking into consideration that caski cells contain HPV whole genome and expresses wild type $\mathrm{p} 53$ while $\mathrm{C} 33 \mathrm{~A}$ is HPV negative and express mutant type p53.

C33AE6 cells are modified C33A cells obtained by stable transfection of the parental C33A cell line with HPV16 E6 cDNA so the cells expresses only HPV E6 oncogene ( partial HPV genome)and contain mutant type p53 the same as C33A cell lines (Hampson et al., 2001) .

Reduction of p53 expression afterTax1BP3 knockout was also confirmed by flow cytometryric showing the same results in all cell lines monoclones (figure 3,4,5).

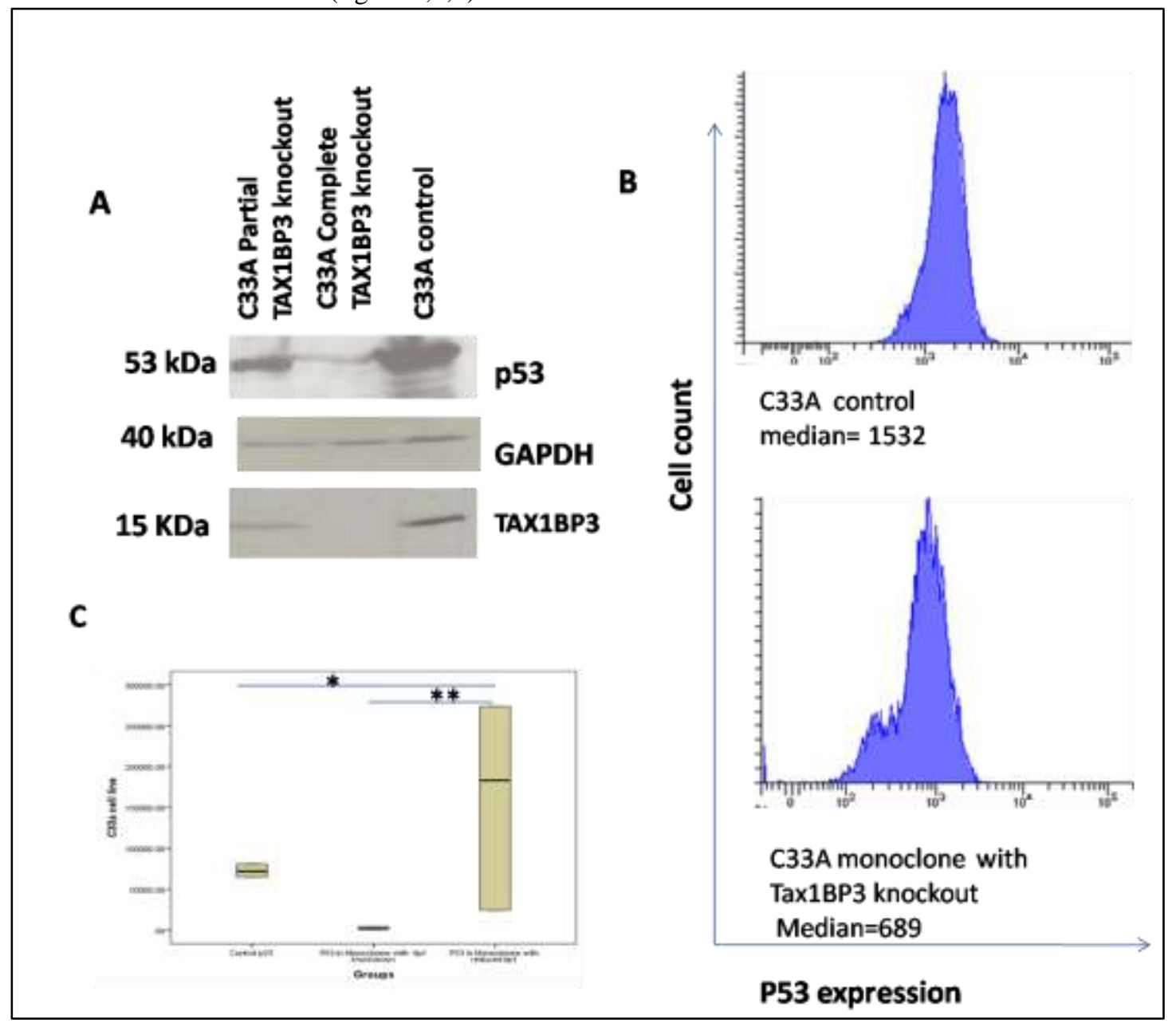

(Fig 3) A) western blot analysis of Tax1BP3 expression of protein extracted from C33A stably transfected monoclones showing the ability of CRISPR/Cas9 to knockout Tax1BP3, both monoclones with complete Tax1BP3 knockout (biallelic) and partial Tax1BP3 knockout (monoallelic) associated with a reduction of p53 expression.

B)Flowcytometric analysis of p53 expression level showing p53 expression reduction in monoclones with CRISPR/Cas mediated Tax1BP3 knockout in comparison to their control. C) Statistical analysis of p53 expression reduction in C33A monoclonesusingKruskal Wallis followed by pairwise comparisons(n=6), monoclones with partial Tax1BP3 Knockoutshow * significance $<0.05$ relative to Control p53 monoclones with complete Tax1BP3 Knockoutshow $^{* *}$ high significance $<0.001$ relative to Control p53. 


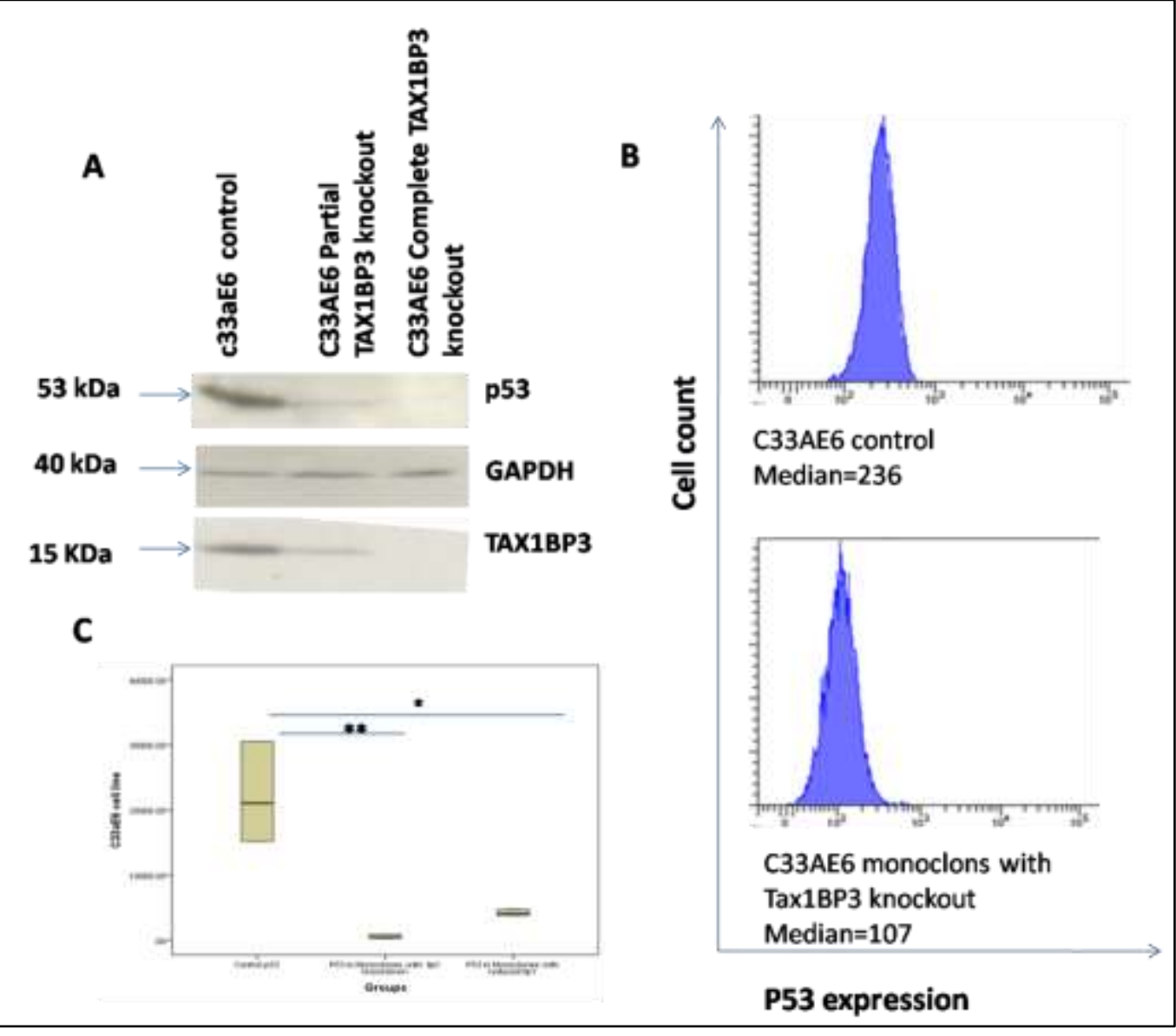

(Fig 4) A) western blot analysis of Tax1BP3 expression of protein extracted from C33AE6 stably transfected monoclones showing the ability of CRISPR/Cas9 to knockout Tax1BP3, both monoclones with complete Tax1BP3 knockout (biallelic) and partial Tax1BP3 knockout (monoallelic) associated with a reduction of p53 expression.

B)Flowcytometric analysis of p53 expression level showing p53 expression reduction in monoclones with CRISPR/Cas mediated Tax1BP3 knockout in comparison to their control. C) Statistical analysis of p53 expression reduction in C33AE6 monoclones using Kruskal Wallis followed by pairwise comparisons(n=6), monoclones with partial Tax1BP3 Knockoutshow ${ }^{*}$ significance $<0.05$ relative to Control p53 monoclones with complete Tax1BP3 Knockoutshow $^{* *}$ high significance $<0.001$ relative to Control p53. 


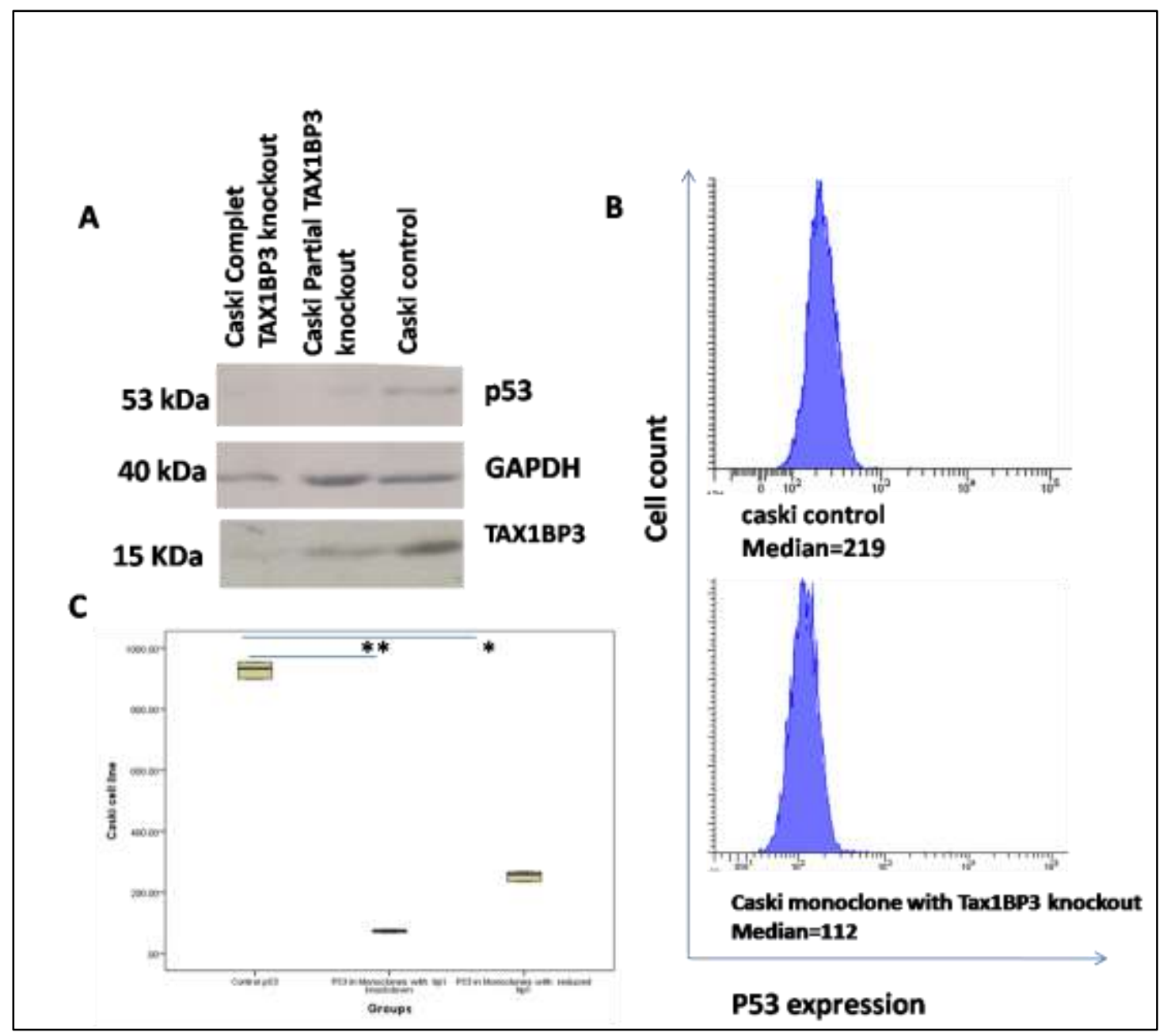

(Fig 5) A) western blot analysis of Tax1BP3 expression of protein extracted from caski stably transfected monoclones showing the abilityof CRISPR/Cas9 to knockout Tax1BP3, both monoclones with complete Tax1BP3 knockout (biallelic) and partial Tax1BP3 knockout (monoallelic) associated with a reduction of p53 expression.

B)Flowcytometric analysis of p53 expression level showing p53 expression reduction in monoclones with CRISPR/Cas mediated Tax1BP3 knockout in comparison to their control. C) Statistical analysis of p53 expression reduction in caskimonoclonesusingKruskal Wallis followed by pairwise comparisons $(n=6)$, monoclones with partial Tax1BP3 Knockoutshow ${ }^{*}$ significance $<0.05$ relative to Control p53 monoclones with complete Tax1BP3 Knockoutshow $^{* *}$ high significance $<0.001$ relative to Control p53.

\section{Discussion:-}

The work presented describes the ability of the bacterial RNA guided endonuclease of CRISPR/Cas9 system to efficiently inactivate Tax1BP3, many previous studies have reported the technology of siRNA or shRNA to knockdown Tax1BP3 (Oliver et al., 2011, Han et al., 2012) however their effect is temporary, counteracted by human cells in long run and cannot be inherited narrowing its application especially in therapeutic purposes (Hu et al., 2014, Xiao-Jie et al., 2015)

Monoclones with reduced Tax1BP3 expression were also obtained which probably result from a monoallelic knockout in this regard the paired-gRNA guided double kinking Cas9 system could be better applied in the future to increase cas9 system specificity and decreases potential off targets (Ran et al., 2013).

The experiment was the extended to analyze the prospective consequence of Tax1BP3 knockout on the expression level of p53 in HPV positive (c33aE6 and caski) and HPV negative cell lines and interestingly after 
Tax1BP3knockdown a significant reduction in p53 expression was detected in all cell lines monoclones taking into consideration that both c33a and c33aE6 contain mutant type p53 while caski cell line contain wild type p53. Reduced Tax1BP3expression (partial knockout) was also associated with a reduction in p53 expression in all monoclones.

Tax1BP3 interacts with many intracellular protein partners such as rhotekin (Reynaud et al., 2000), glutaminase L (Olalla et al., 2001), potassium channel Kir2.3 (Alewine et al., 2006), $\beta$ catenin (Katoh et al., 2007), HPV16 E6 (Hampson et al., 2004), Rho guanine exchange factor 16 (Oliver et al., 2011), plexinD1 and AT hook DNA binding containing 1 (AHDC1) proteins (Shalaby et al., 2011), Of particular interest to note that Tax1BP3 was documented to inhibits the proliferation of colon cancer cells suggesting that Tax1BP3 might function as a tumor suppressor and on the other hand, elevated Tax1BP3 expression levels was detected in human invasive breast cancer (Han et al., 2012) and human glioblastoma and was associated with advanced stages and poor prognosis revealed an oncogenic function of Tax1BP3 (Wang et al., 2014).

What is the relationship between $\mathrm{p} 53$ and Tax1BP3?

P53 normally expressed in low level due to its interaction with Murine Double Minute 2(MDM2) which act as E3 ubiquitin ligase promoting the proteasome mediated p53 protein degradation ( $\mathrm{Gu}$ et al., 2006). p53 is also regulated by ARF which activates p53 by inhibiting MDM2 mediated p53 degradation. In a previous study on glioma cells, Tax1BP3 was shown to enhance the protein interaction between LZAP and ARF leading to polyubiquitination and degradation of p53(Han et al., 2012).

However Opposite to those observations another study on osteosarcoma cell line demonstrated that interaction between LZAP and ARF leading to maintaining p53 stability and increased p53 transcriptional activity (Wang et al., 2006). The mechanism by which Tax1BP3 affect the expression of p53 is not completely understood., Taking together, we speculate that Tax1BP3 might regulate p53 expression through its interaction with LZAP protecting p53 from degradation and might considered as one explanation for reduced p53 expression after Tax1BP3knockout . It is noteworthy that previous work has shown Direct evidence between $\beta$-catenin and p53 demonstrating that excess $\beta$-catenin can induce an accumulation of active p53 which explain, at least in part, loss of p53 activity in tumors harboring deregulated $\beta$-catenin, such as colorectal cancer and hepatocellular carcinoma (Han et al., 2012) taking those together with the fact that Tax1BP3interact with beta- catenin and inhibit its transcriptional activity(Lowy et al., 2006) thus we hypothesise that Tax1BP3 knockout may associated with down regulation of beta-catenin and consequently p53 expression.

We noted that other report showed that Tax1BP3 knockdown remarkably change the cellular morphologyin gliomacells(Wang et al., 2014). However, we did not see any cellular morphology difference in all monoclones after Tax1BP3knockdown in comparison to the original cell line or the scramble control. This difference might be due to the different cellular context between glioblastoma cell and cervical cancer cells.

As Tax1BP3interact with several intracellular proteins, we believe that it would be interesting to investigate whether Tax1BP3 knockout can affect the expression level of any other Tax1BP3 partners especially beta-catenin and LZAP and may help to understand the exact mechanism by which Tax1BP3 could affect the expression of p53.

\section{Conclusion:-}

the presented data showed that reduced levels of Tax1BP3 directly correlated with reduced levels of p53 protein expression. This effect was seen in cells whether or not they expressed mutant (C33A) or wild type (CaSki) p53 or full or partial HPV genome (CaSki and C33AE6). These results suggest that Tax1BP3 regulates p53 protein levels in HPV positive and negative cells by a novel and non-canonical means.

\section{Acknowledgment:-}

This work was supported by Egypt - UK Newton Musharafa fund. 


\section{References:-}

1. Ainsworth, J., Thomas, M., Banks, L., Coutlee, F., \&Matlashewski, G. (2008). Comparison of p53 and the PDZ domain containing protein MAGI-3 regulation by the E6 protein from high-risk human papillomaviruses. Virology journal, 5(1), 67.

2. Alewine, C., Olsen, O., Wade, J. B., \& Welling, P. A. (2006). TIP-1 has PDZ scaffold antagonist activity. Molecular biology of the cell, 17(10), 4200-4211.

3. Cong, L., Ran, F. A., Cox, D., Lin, S., Barretto, R., Habib, N., ...\& Zhang, F. (2013). Multiplex genome engineering using CRISPR/Cas systems. Science, 339(6121), 819-823.

4. Ebina, H., Misawa, N., Kanemura, Y., \&Koyanagi, Y. (2013). Harnessing the CRISPR/Cas9 system to disrupt latent HIV-1 provirus. Scientific reports, 3 .

5. Gaj, T., Gersbach, C. A., \&Barbas, C. F. (2013). ZFN, TALEN, and CRISPR/Cas-based methods for genome engineering. Trends in biotechnology, 31(7), 397-405.

6. Gu, B., \& Zhu, W. G. (2012). Surf the post-translational modification network of p53 regulation. International journal of biological sciences, 8(5), 672.

7. Wang, J., He, X., Luo, Y., \& Yarbrough, W. G. (2006). A novel ARF-binding protein (LZAP) alters ARF regulation of HDM2. Biochemical Journal, 393(2), 489-501.

8. Hampson, L., Li, C., Oliver, A. W., Kitchener, H. C., \&Hampson, I. N. (2004). The PDZ protein Tip-1 is a gain of function target of the HPV16 E6 oncoprotein. International journal of oncology, 25(5), 1249-1256.

9. Han, M., Wang, H., Zhang, H. T., \& Han, Z. (2012). The PDZ protein TIP-1 facilitates cell migration and pulmonary metastasis of human invasive breast cancer cells in athymic mice. Biochemical and biophysical research communications, 422(1), 139-145.

10. Hu, W., Kaminski, R., Yang, F., Zhang, Y., Cosentino, L., Li, F., ...\& Mo, X. (2014). RNA-directed gene editing specifically eradicates latent and prevents new HIV-1 infection. Proceedings of the National Academy of Sciences, 111(31), 11461-11466..

11. Kanamori, M., Sandy, P., Marzinotto, S., Benetti, R., Kai, C., Hayashizaki, Y., ...\& Suzuki, H. (2003). The PDZ protein tax-interacting protein-1 inhibits $\beta$-catenin transcriptional activity and growth of colorectal cancer cells. Journal of Biological Chemistry, 278(40), 38758-38764.

12. Katoh, M., \&Katoh, M. (2007). WNT signaling pathway and stem cell signaling network. Clinical cancer research, 13(14), 4042-4045.

13. Lin, S. R., Yang, H. C., Kuo, Y. T., Liu, C. J., Yang, T. Y., Sung, K. C., ... \& Wu, F. Y. (2014). The CRISPR/Cas9 system facilitates clearance of the intrahepatic HBV templates in vivo. Molecular TherapyNucleic Acids, 3, e186.

14. Lowy, A. M., Clements, W. M., Bishop, J., Kong, L., Bonney, T., Sisco, K., ...\&Groden, J. (2006). $\beta$ Catenin/Wnt signaling regulates expression of the membrane type 3 matrix metalloproteinase in gastric cancer. Cancer research, 66(9), 4734-4741.

15. Mali, P., Yang, L., Esvelt, K. M., Aach, J., Guell, M., DiCarlo, J. E., ...\& Church, G. M. (2013). RNA-guided human genome engineering via Cas9. Science, 339(6121), 823-826.

16. Mohanty, S., Ovee, M., \& Banerjee, M. (2015). pdz domain recognition: insight from human tax-interacting protein 1 (TIP-1) interaction with target proteins. Biology, 4(1), 88-103.

17. Olalla, L., Aledo, J. C., Bannenberg, G., \&Márquez, J. (2001). The C-terminus of human glutaminase L mediates association with PDZ domain-containing proteins 1. FEBS letters, 488(3), 116-122.

18. Oliver, A. W., He, X., Borthwick, K., Donne, A. J., Hampson, L., \&Hampson, I. N. (2011). The HPV16 E6 binding protein Tip-1 interacts with ARHGEF16, which activates Cdc42. British journal of cancer, 104(2), 324.

19. Ran, F. A., Hsu, P. D., Lin, C. Y., Gootenberg, J. S., Konermann, S., Trevino, A. E., ... \& Zhang, F. (2013). Double nicking by RNA-guided CRISPR Cas9 for enhanced genome editing specificity. Cell, 154(6), 13801389.

20. Reynaud, C., Fabre, S., \&Jalinot, P. (2000). The PDZ protein TIP-1 interacts with the Rho effector rhotekin and is involved in Rho signaling to the serum response element. Journal of Biological Chemistry, 275(43), 3396233968.

21. Rousset, R., Fabre, S., Desbois, C., Bantignies, F., \&Jalinot, P. (1998). The C-terminus of the HTLV-1 Tax oncoprotein mediates interaction with the PDZ domain of cellular proteins. Oncogene, 16(5).

22. Shalaby, M. A., Hampson, L., Oliver, A., \&Hampson, I. (2011). Identification of PlexinD1 and AHDC1 as a putative interactors for Tip-1 protein. Genes \& Genomics, 33(4), 399-405.

23. Wang, H., Han, M., WhetsellJr, W., Wang, J., Rich, J., Hallahan, D., \& Han, Z. (2014). Tax-interacting protein 1 coordinates the spatiotemporal activation of Rho GTPases and regulates the infiltrative growth of human glioblastoma. Oncogene, 33(12), 1558. 
24. Xiao-Jie, L., Hui-Ying, X., Zun-Ping, K., Jin-Lian, C., \& Li-Juan, J. (2015). CRISPR-Cas9: a new and promising player in gene therapy. Journal of medical genetics, jmedgenet-2014.

25. Yan, H., Kapoor, V., Nguyen, K., Akers, W. J., Li, H., Scott, J., ... \&Hallahan, D. (2016). Anti-tax interacting protein-1 (TIP-1) monoclonal antibody targets human cancers. Oncotarget, 7(28), 43352.

26. Zencir, S., Banerjee, M., Dobson, M. J., Ayaydin, F., Fodor, E. A., Topcu, Z., \&Mohanty, S. (2013). New partner proteins containing novel internal recognition motif for human glutaminase interacting protein (hGIP). Biochemical and biophysical research communications, 432(1), 10-15.

27. Zhen, S., Hua, L., Takahashi, Y., Narita, S., Liu, Y. H., \& Li, Y. (2014). In vitro and in vivo growth suppression of human papillomavirus 16-positive cervical cancer cells by CRISPR/Cas9. Biochemical and biophysical research communications, 450(4), 1422-1426. 